\title{
Gravitational waves from pulsating stars: Evolving the perturbation equations for a relativistic star
}

\author{
Gabrielle Allen, ${ }^{1,2}$ Nils Andersson, ${ }^{3,4}$ Kostas D. Kokkotas, ${ }^{1,5}$ and Bernard F. Schutz ${ }^{1,2}$ \\ ${ }^{1}$ Max Planck Institute for Gravitational Physics, The Albert Einstein Institute, D-14473 Potsdam, Germany \\ ${ }^{2}$ Department of Physics and Astronomy, University of Wales College of Cardiff, Cardiff CF2 3YB, United Kingdom \\ ${ }^{3}$ Department of Physics, Washington University, St Louis, Missouri 63130 \\ ${ }^{4}$ Institut für Astronomie und Astrophysik, Universität Tübingen, D-72076 Tübingen, Germany \\ ${ }^{5}$ Department of Physics, Aristotle University of Thessaloniki, Thessaloniki 54006, Greece
}

(Received 8 April 1997; published 17 November 1998)

\begin{abstract}
We consider the perturbations of a relativistic star as an initial-value problem. Having discussed the formulation of the problem (the perturbation equations and the appropriate boundary conditions at the center and the surface of the star) in detail, we evolve the equations numerically from several different sets of initial data. In all the considered cases, we find that the resulting gravitational waves carry the signature of several of the star's pulsation modes. Typically, the fluid $f$ mode, the first two $p$ modes, and the slowest damped gravitational $w$ mode are present in the signal. If such mode signals, from coalescing neutron stars or following a supernova, can be detected by future gravitational-wave antennae, one can hope to infer detailed information about neutron stars. Since a perturbation evolution should adequately describe the late time behavior of a dynamically excited neutron star, the present work can also be used as a benchmark test for future fully nonlinear simulations. [S0556-2821(98)01120-5]
\end{abstract}

PACS number(s): 04.40.Dg, 04.30.-w, 95.30.Sf, 97.60.Jd

\section{INTRODUCTION}

It is well known that a neutron star has a rich pulsation spectrum [1-4]. We expect the stellar pulsation modes to be excited in many neutron star processes (ranging from core quakes to the formation of a neutron star through gravitational collapse). A signal carrying the signature of these modes could, if detected by future gravitational-wave antennae, provide useful information about the star. The question is whether the various stellar pulsation modes can be dynamically excited to a level that makes them astrophysically relevant. This question is especially interesting because of the existence of "spacetime" modes [5], which have no analogue in the Newtonian theory of stellar pulsation. It has been shown $[6,7]$ that these modes, together with the normal fluid pulsation modes, can provide valuable information about the mass, size, and equation of state of neutron stars. Much of the initial deformation of spacetime in, e.g., a supernova collapse could conceivably be released through spacetime pulsation modes, yet most studies of neutron star formation and pulsation have not treated them because they have been done within the Newtonian or post-Newtonian approximations.

With the present paper, we take the first steps towards an answer to the question of mode-excitation. We describe numerical evolutions of the equations that describe a perturbed relativistic star for various sets of initial data. Evolutions of the perturbation equations for black holes have provided interesting information (see [8] for references), and it seems likely that this approach should prove equally instructive for stars. Our main focus is on the evolution problem itself: We formulate the problem and discuss all relevant equations in some detail. We also present results from two test problems, which indicate that for a range of initial conditions, the energy emitted is shared broadly among the pulsation and spacetime modes. This argues strongly, if in a preliminary way, that dynamical calculations of the collision of two neutron stars or the late stages of a gravitational collapse done within the Newtonian and post-Newtonian approximations may seriously underestimate the amount and the spectral character of the emitted gravitational radiation. (In order to avoid confusion, we should emphasize that the postNewtonian picture is still an excellent description of the binary inspiral phase.)

The plan of the paper is as follows. In Sec. II we formulate the linear perturbation problem for a neutron star in general relativity as an initial-value problem, with special attention to the boundary conditions and the treatment of the center of the star. In Sec. III we solve the initial-value problem numerically for two sets of initial conditions, which represent in some sense the extremes of the balance between exciting fluid modes on the one hand and spacetime modes on the other. The spectrum of the gravitational waves generated from these initial data show clearly the signatures of both the fluid modes and the spacetime modes. Importantly, in both sets the excitation of the spacetime modes is appreciable. In Sec. IV we discuss the significance of the results, and our plans for future work to determine a more realistic initial-data set to use for such calculations. We begin by providing, in the remainder of Sec. I, a more detailed motivation for this work in the context of numerical relativity and gravitational wave detection, and a brief background on relativistic stellar pulsation theory with special focus on recent developments. 


\section{A. Gravitational-wave astronomy and numerical relativity}

With the building of several large-scale laser interferometers for gravitational-wave detection well under way $[9,10]$ "gravitational-wave astronomy" may be established around the turn of the century. But many fundamental problems remain to be solved before this goal can be reached. For the theorist, the most pressing problems concern modeling the processes which generate gravitational waves [11]. Only by comparing observational data to such models can we hope to infer detailed information about the various gravitationalwave sources.

Simulations of processes involving neutron stars have (with a few exceptions) so far used Newtonian gravity [12], see the discussion in Sec. III C. This is mainly due to the difficulties of consistently treating both the matter and the gravitational field within the framework of the standard Arnowitt-Deser-Misner (ADM) formalism. In these Newtonian simulations, the gravitational radiation is calculated using the quadrupole formula. That is, gravitational waves originate solely from stellar fluid motion-there is no contribution from the dynamics of the gravitational field itself. However, the dynamics of the gravitational field should also contribute to the emerging gravitational radiation. This is clear from the black hole case, in which the quasinormalmode oscillations are entirely due to the dynamical spacetime [8]. At an intuitive level, one would expect similar features to exist for stars, although they are probably less dominant, since a neutron star is less relativistic than a black hole. As we describe below this is, indeed, the case. There is a set of pulsation modes of a relativistic star that can be directly associated with the spacetime curvature. But it is not yet known how important the spacetime contribution to the gravitational-wave emission will be in a dynamical scenario.

There is growing optimism in the numerical relativity community over the feasibility of simulating neutron stars within general relativity, but even though such projects are ultimately the only way to provide reliable gravitationalwave estimates, the first results will not be available for some years. In the meantime, we must resort to approximate methods. The present paper describes a project to investigate the excitation of the stellar pulsation modes using perturbation theory. We recall that non-linear numerical calculations for black holes have shown that the quasinormal modes tend to be clearly present in the radiation. Furthermore, comparisons with non-linear calculations have indicated that the more tractable linear approximations can provide accurate waveforms for a wide range of initial data. A particularly interesting example of this is the "close-limit" approximation for colliding black holes [13]. It seems likely that perturbation calculations will also be a useful tool for problems involving relativistic stars. The relative simplicity of the perturbation approach compared to the full non-linear calculations makes its application and interpretation easier. We can hope to learn some of the relevant physics by pursuing the perturbation approach. In the near future, while non-linear codes are still being developed, approximate studies can lead the way. A further benefit from this work is its application as a test for the full non-linear codes for simulations of stellar processes.

\section{B. Pulsating stars}

The study of stellar pulsation in a general relativistic context has a considerable history, dating back to the work of Thorne and his colleagues in the late 1960's [14] (for a recent review see [15]). Originally, the stellar pulsation problem was approached from a "Newtonian" viewpoint. In this picture, motion in the stellar fluid generates gravitational waves which carry energy away from the system. The characteristic frequencies of the various pulsation modes of the star thus become complex valued. Due to the weak coupling between matter and gravitational waves, the damping rate of a typical pulsation mode is very low [16].

This "Newtonian" picture is limited in that the dynamical properties of the spacetime itself are neglected. That a dynamic spacetime can add new features to the pulsation problem can be understood in terms of a simple, but instructive, model problem due to Kokkotas and Schutz [17]. There are pulsation modes directly associated with spacetime itself. These new modes have been termed "gravitational-wave modes" (or $w$-modes) [5]. They have relatively high oscillation frequencies $(6-14 \mathrm{kHz}$ for typical neutron stars) and barely excite any fluid motion. They are also rapidly damped, with a typical lifetime of a fraction of a millisecond. Our understanding of the $w$-modes has improved with a body of recent work $[4,18-20]$.

The existence of pulsation modes that are directly associated with the spacetime itself is interesting from a theoretical point of view, but it is necessary to establish if these modes are of astrophysical significance [6]. One can argue that the gravitational-wave modes could be relevant in many scenarios. Consider, for example, gravitational collapse to a neutron star, or the coalescence of two neutron stars. In both cases will there be changes in the deformation of spacetime, which could potentially lead to considerable amounts of energy being radiated through the $w$-modes. Detailed calculations are needed to provide quantitative information. In essence, there are two important questions that must addressed: (i) Will the gravitational-wave modes be excited in processes such as supernova collapse or neutron star coalescence? (ii) What is the possibility of observing such modes in data from the new generation of gravitational-wave detectors, and what physical information could such observations provide?

As an initial attempt to answer the second question was recently provided by two of us [7]. As for the first issue, we have studied scattering of wave-packets by a uniform density star [6]. This is not a problem of great astrophysical importance (it is difficult to imagine a situation, where the impinging gravitational wave has sufficient magnitude to make the scattered wave observable), but the results were nevertheless encouraging. The resultant gravitational waves show the clear signature of the $w$-modes for neutron star sized objects $(R / M \approx 5)$. Similar results have since been obtained for a particle falling in the stellar spacetime [21]. The present paper describes more detailed work along these lines: We evolve the linearized equations from general initial data. Most importantly, our analysis is not restricted to an initial perturbation with compact support in the vacuum outside the star. To construct our stellar model, we use a polytropic 
equation of state. Contrary to the simple uniform density model, polytropes allow several fluid pulsation modes to exist (the single $f$-mode and an infinite sequence of $p$-modes).

\section{STELLAR PERTURBATIONS AS AN INITIAL VALUE PROBLEM}

In this section we describe the stellar perturbation problem. We will introduce all the necessary equations, but not discuss their origin in great detail. For further details we refer the reader to previous work $[14,16,22-24]$. It is relevant to point out that most of the existing work on perturbations of stars has been performed in the frequency domain (after Fourier decomposition of the various perturbed quantities). This is the natural approach as long as one is mainly interested in the spectral properties of a star. Kind, Ehlers and Schmidt [25] appear to be the only authors that have considered relativistic stellar perturbations as an initial value problem. (Perturbations of a time-dependent geometry were considered by Seidel et al. [26-28] in the context of gravitational collapse.) The motivation of Kind et al. was to show that initial Cauchy data with the appropriate junction conditions at the stellar surface determines a unique solution to the time-dependent equations. Thus, Kind et al. address important mathematical questions for the stellar evolution problem. The motivation of the present investigation is rather different, but our formulation of the problem is still inspired by [25].

\section{A. The stellar model}

A static spherically symmetric stellar model can be described by the metric

$$
d s^{2}=-e^{\nu} d t^{2}+e^{\lambda} d r^{2}+r^{2}\left(d \theta^{2}+\sin ^{2} \theta d \varphi^{2}\right),
$$

where the metric coefficients $\nu$ and $\lambda$ are functions of $r$ only. Specifically,

$$
e^{\lambda}=\left(1-\frac{2 m(r)}{r}\right)^{-1}
$$

and the "mass inside radius $r$ " is represented by

$$
m(r)=4 \pi \int_{0}^{r} \rho r^{2} d r
$$

This means that the total mass of the star is $M=m(R)$, with $R$ being the stars radius. (We use geometric units $G=c=1$ throughout this paper.)

A star in hydrostatic equilibrium is governed by the Tolman-Oppenheimer-Volkoff (TOV) equations: To determine a stellar model, we must solve

$$
\frac{d p}{d r}=-\frac{\rho+p}{2} \frac{d \nu}{d r}
$$

where

$$
\frac{d \nu}{d r}=\frac{2 e^{\lambda}\left(m+4 \pi p r^{3}\right)}{r^{2}} .
$$

These equations do, of course, require an equation of state $p=p(\rho)$ as input. In this paper we consider (for reasons of simplicity) only polytropic equations of state

$$
p=\kappa \rho^{\gamma} .
$$

In particular, we present numerical results for a model with $\kappa=100 \mathrm{~km}^{2}$ and $\gamma=2$. We have performed calculations using different parameters (and also tested our numerical code with some realistic equations of state), but, since the results were similar to those discussed in Sec. III B, we will only discuss one stellar model in detail. The specific model we have chosen has a central density $\rho_{c}=3 \times 10^{15} \mathrm{~g} / \mathrm{cm}^{3}$. The corresponding radius and mass are $R=8.86 \mathrm{~km}$ and $M$ $=1.87 \mathrm{~km} \approx 1.2 M_{\odot}$, respectively. The pulsation properties of this stellar model have already been investigated in detail [5].

\section{B. The perturbation problem}

A general description of the stellar perturbation problem is as follows: We want to solve the perturbed Einstein equations

$$
\delta G_{\mu \nu}=8 \pi \delta T_{\mu \nu} .
$$

The small amplitude motion that a perturbation induces in the stellar fluid is described by displacements $\xi^{r}, \xi^{\theta}$ and $\xi^{\varphi}$. The fluid displacement affects also the pressure and the density of the fluid. To describe these, we use the Eulerian variations $\delta p$ and $\delta \rho$.

As is familiar from black-hole problems [8,29], the perturbed metric can be split into two classes: axial and polar (alternatively called odd and even parity, or toroidal and spheroidal) perturbations. That is, the metric can be written

$$
g_{\mu \nu}=g_{\mu \nu}^{\text {background }}+h_{\mu \nu}^{\text {polar }}+h_{\mu \nu}^{\text {axial }} .
$$

If we work in Regge-Wheeler gauge (which has been used for most of the previous studies of the stellar problem), the axial perturbations are described by four variables linked to the metric coefficients $h_{t \theta}, h_{t \varphi}, h_{r \varphi}$ and $h_{r \theta}$. These perturbations induce a differential rotation of the star, where the only non-zero component of the fluid-displacement vector is $\xi^{\varphi}$. The polar metric perturbations correspond to six variables related to the metric coefficients $h_{t t}, h_{t r}, h_{r r} h_{\theta \theta}, h_{\theta \varphi}$ and $h_{\varphi \varphi}$ together with the fluid displacements $\xi^{r}$ and $\xi^{\theta}$. Exactly as for black holes, it turns out that the equations that govern the axial and the polar quantities decouple in the case 
of a nonrotating star (this will not be the case if the star is rotating $[24,30])$. We thus have two problems that can be approached separately.

In this paper we only consider the polar problem. There are several reasons for this. We have recently considered the initial-value problem for axial perturbations of a uniform density star [6]. Because an axial perturbation does not induce pulsations in the stellar fluid [14], we do not expect the results for a more realistic stellar model to be dramatically different from those for uniform density. Basically, an axial perturbation can only result in the excitation of $w$-modes, and as long as the two stars have similar compactness $(R / M)$, the corresponding frequencies are quite similar for a uniform density star and a more realistic model [4]. Hence, we focus on the polar problem here.

The polar problem corresponds to seven perturbed field equations and three equations of motion for four metric perturbations and four fluid variables [14,24,25]. At first this would seem to be an overdetermined problem, but this is not the case. Three of our original equations (the equations of motion, say) are void of new information because of the Bianchi identities. Moreover, we can use

$$
\delta \rho=\frac{1}{C_{s}^{2}} \delta p,
$$

where $C_{S}$ is the acoustic wave-speed in the stellar fluid. We are thus left with seven equations for seven unknown variables.

\section{Evolution equations}

There are many possible formulations of the perturbation problem, even within the Regge-Wheeler gauge, and it is difficult to predict if a specific one may be advantageous from the point of view of numerical evolutions. Here we have chosen a formulation that reflects the physics of the system. That is, we consider a set of wave-equations describing gravitational waves coupled to acoustic waves in the stellar fluid. Furthermore, we have cast the "spacetime" equations into a form which resembles the more familiar equations for black-hole perturbations. This facilitates a comparison between the two problems.

The perturbed Einstein equations and the fluid equations of motion can be manipulated into a set of evolution equations for a reduced number of variables. The remaining spacetime and fluid perturbations are determined (at all times) by a number of constraint equations. Here we evolve two spacetime variables $F(t, r)$ and $S(t, r)$ which are related to the metric perturbations by

$$
\begin{gathered}
h_{\theta \theta}(t, r)=r F(t, r), \\
h_{t t}(t, r)-\frac{e^{\nu}}{r^{2}} h_{\theta \theta}(t, r)=r S(t, r) .
\end{gathered}
$$

These functions are related to the standard Regge-Wheeler functions $K(t, r)$ and $H_{0}(t, r)$ (see, e.g., [14] or [24]) by $F$ $=r K, S=e^{\nu}\left(H_{0}-K\right) / r$. We also evolve a fluid function (the perturbed relativistic enthalpy)

$$
H(t, r)=\frac{\delta p}{\rho+p},
$$

which is defined only inside the star. In these definitions of $F, S$ and $H$, we have suppressed the angular dependence [31].

The above variables are not obvious, but we have good reasons for introducing them. Let us first consider the function $S$. When we introduce this function, and also use the standard definition of the "tortoise coordinate",

$$
\frac{\partial}{\partial r_{*}}=e^{(\nu-\lambda) / 2} \frac{\partial}{\partial r}
$$

we find that [32]

$$
\begin{gathered}
-\frac{\partial^{2} S}{\partial t^{2}}+\frac{\partial^{2} S}{\partial r_{*}^{2}}+\frac{2 e^{\nu}}{r^{3}}\left[2 \pi r^{3}(\rho+3 p)+m-(n+1) r\right] S \\
=-\frac{4 e^{2 \nu}}{r^{5}}\left(\frac{\left(m+4 \pi p r^{3}\right)^{2}}{(r-2 m)}+4 \pi \rho r^{3}-3 m\right) F,
\end{gathered}
$$

where $2 n=(l-1)(l+2)$. We thus have a simple waveequation with no explicit dependency on the perturbations of the fluid. For the other "metric" variable $F$, we find [32]

$$
\begin{aligned}
-\frac{\partial^{2} F}{\partial t^{2}} & +\frac{\partial^{2} F}{\partial r_{*}^{2}}+\frac{2 e^{\nu}}{r^{3}}\left[2 \pi r^{3}(3 \rho+p)+m-(n+1) r\right] F \\
= & -2\left[4 \pi r^{2}(p+\rho)-e^{-\lambda}\right] S \\
& +8 \pi(\rho+p) r e^{\nu}\left(1-\frac{1}{C_{s}^{2}}\right) H .
\end{aligned}
$$

This equation is also quite simple, but here the coupling to the fluid variable $H$ is apparent. Both of our wave equations are valid inside the star as well as in the exterior vacuum. In the exterior the mass $m$ is, obviously, the total mass $M$. Our reason for introducing $S$ and $F$ should now be apparent: In terms of these variables, the perturbation equations become similar to the wave-equation that governs a perturbed Schwarzschild black hole [8], or the equation for axial perturbations of a star [33]. That the polar equations can be cast in a similar form has not been shown previously.

The equation that governs the fluid variable $H$ is, however, still somewhat messy. After some algebra, we find that [32] 


$$
\begin{aligned}
-\frac{1}{C_{s}^{2}} & \frac{\partial^{2} H}{\partial t^{2}}+\frac{\partial^{2} H}{\partial r_{*}^{2}}+\frac{e^{(\nu+\lambda) / 2}}{r^{2}}\left[\left(m+4 \pi p r^{3}\right)\left(1-\frac{1}{C_{s}^{2}}\right)+2(r-2 m)\right] \frac{\partial H}{\partial r_{*}}+\frac{2 e^{\nu}}{r^{2}}\left[2 \pi r^{2}(\rho+p)\left(3+\frac{1}{C_{s}^{2}}\right)-(n+1)\right] H \\
= & \left(m+4 \pi p r^{3}\right)\left(1-\frac{1}{C_{s}^{2}}\right) \frac{e^{(\lambda-\nu) / 2}}{2 r}\left(\frac{e^{\nu}}{r^{2}} \frac{\partial F}{\partial r_{*}}-\frac{\partial S}{\partial r_{*}}\right)+\left[\frac{\left(m+4 \pi p r^{3}\right)^{2}}{r^{2}(r-2 m)}\left(1+\frac{1}{C_{s}^{2}}\right)-\frac{m+4 \pi p r^{3}}{2 r^{2}}\left(1-\frac{1}{C_{s}^{2}}\right)\right. \\
& -4 \pi r(3 p+\rho)] S+\frac{e^{\nu}}{r^{2}}\left[\frac{2\left(m+4 \pi p r^{3}\right)^{2}}{r^{2}(r-2 m)} \frac{1}{C_{s}^{2}}-\frac{m+4 \pi p r^{3}}{2 r^{2}}\left(1-\frac{1}{C_{s}^{2}}\right)-4 \pi r(3 p+\rho)\right] F .
\end{aligned}
$$

Finally, we have the Hamiltonian constraint [32]

$$
\begin{aligned}
\mathcal{H}:= & \frac{\partial^{2} F}{\partial r_{*}^{2}}-\frac{e^{(\nu+\lambda) / 2}}{r^{2}}\left(m+4 \pi r^{3} p\right) \frac{\partial F}{\partial r_{*}}+\frac{e^{\nu}}{r^{3}}\left[12 \pi r^{3} \rho-m-2(n+1) r\right] F-r e^{-(\nu+\lambda) / 2} \frac{\partial S}{\partial r_{*}} \\
& +\left(8 \pi r^{2}(\rho+p)-(n+3)+\frac{4 m}{r}\right) S+\frac{8 \pi r}{C_{s}^{2}} e^{\nu}(\rho+p) H=0,
\end{aligned}
$$

which must be satisfied by the initial data and also throughout an evolution. We will discuss this constraint in more detail in Sec. III A.

Here it could be worthwhile to note that it is possible to find a new "fluid" variable such that the corresponding wave equation [that would replace Eq. (16)] contains no first derivatives. However, this extra step is not convenient because it involves the solution of a differential equation involving $C_{s}^{2}$.

It is also meaningful to comment on the number of equations that we use. It is well known that the interior problem for polar perturbations can be reduced to two coupled wave equations [23,24]. It would be easy to use Eq. (17) to replace $H$ in Eq. (15) (as was done by Ipser and Price [23] and Kojima [24]). If we do this, we get

$$
\begin{gathered}
-\frac{1}{C_{s}^{2}} \frac{\partial^{2} F}{\partial t^{2}}+\frac{\partial^{2} F}{\partial r_{*}^{2}}-\left(1-\frac{1}{C_{s}^{2}}\right) \frac{e^{(\nu+\lambda) / 2}}{r^{2}}\left(m+4 \pi p r^{3}\right) \frac{\partial F}{\partial r_{*}}+\frac{e^{\nu}}{r^{3}}\left[4 \pi r^{3}\left(3 \rho+\frac{p}{C_{s}^{2}}\right)-m\left(1-\frac{3}{C_{s}^{2}}\right)-2(n+1) r\right] F \\
=\left(1-\frac{1}{C_{s}^{2}}\right) r e^{-(\nu+\lambda) / 2} \frac{\partial S}{\partial r_{*}}+\left[2 e^{-\lambda}+\left(1-\frac{1}{C_{s}^{2}}\right)(n+1)-8 \pi(p+\rho) r^{2}\right] S .
\end{gathered}
$$

However, Eq. (18) is rather "unphysical" because a metric variable $(F)$ plays the role of the fluid (in the sense that it is governed by the sound speed $C_{s}$ ). Beside, it is useful to keep one fluid variable explicit. With the equations for $S, F$, and $H$ above, we can easily monitor the fluid motion during the evolution. Moreover, Kind et al. [25] have shown that, as long as $\rho>0$ at the surface, our problem is well posed (that a unique solutions exists). The result of Kind et al. is applicable also to our evolutions even though our density distribution vanishes as $r \rightarrow R$, since we always introduce the "surface" of the star at a small nonzero value of $\rho$ in the numerical code.

Let us now turn to the exterior problem. In the vacuum outside the star, the problem is identical to that for a perturbed Schwarzschild black hole. Thus, the equations simplify considerably, and we only need to consider the two equations for $S$ and $F$ (note that the two effective potentials are equal in the exterior vacuum). Furthermore, we know from the studies of perturbed black holes that we can reduce the problem even further. We only need to consider one single homogeneous wave equation-the Zerilli equation [8]. If we define $[28,34]$

$$
\frac{(n+1)(n r+3 M)}{r^{3}} Z=-\frac{1}{r} \frac{\partial F}{\partial r_{*}}+\frac{(n+2) r-M}{r^{3}} F+S,
$$

we find that $Z$ evolves according to

$$
\frac{\partial^{2} Z}{\partial t^{2}}-\frac{\partial^{2} Z}{\partial r_{*}^{2}}+V_{Z}(r) Z=0
$$

where the effective potential is given by

$$
\begin{aligned}
V_{Z}(r)= & \frac{2 e^{\nu}}{(n r+3 M)^{2} r^{3}} \\
& \times\left[n^{2}(n+1) r^{3}+3 n^{2} M r^{2}+9 n M^{2} r+9 M^{3}\right] .
\end{aligned}
$$


This equation has the advantage that the function $Z$ is gauge-invariant and unconstrained $[28,34]$. But from the point of view of a numerical evolution, it may be preferable to solve the coupled system for $S$ and $F$ - the variables that are used inside the star-rather than switching to the Zerilli equation in the exterior. Furthermore, it turns out that it is trivial to obtain gauge-invariant (but constrained) quantities from $S$ and $F$. If we use $q_{1}$ and $q_{2}$ to denote the gauge invariant variables that were first introduced by Seidel [28], we find that

$$
\begin{aligned}
& q_{1}=H_{0}=\frac{F}{r}+r e^{-\nu} S, \\
& q_{2}=K=\frac{F}{r} .
\end{aligned}
$$

These equations prescribe how the gauge invariant quantities can be generated from the specific functions that we solve for in Regge-Wheeler gauge. It is straightforward to generate $q_{1}$ and $q_{2}$ once we know $S$ and $F$ (or the standard ReggeWheeler functions $H_{0}$ and $K$ ). Furthermore, for the fluid variable one finds that $\delta \rho$ in Regge-Wheeler gauge corresponds immediately to the gauge-invariant variable $\Delta \eta$ that was used by Seidel [28].

\section{Behavior at the center and the surface of the star}

When evolving the perturbation equations, one must give special consideration to the behavior of the variables close to the center and the surface of the star.

To infer the behavior at the center is relatively easy because we know that, for a physical solution, all the perturbed variables should be regular at $r=0$. Working in the Fourier domain and expanding all variables in powers of $r$ (as was done by Lindblom and Detweiler [16]), we can infer that

$$
\left.\begin{array}{l}
F \sim r^{l+1} \\
S \sim r^{l+1} \\
H \sim r^{l}
\end{array}\right\} \text { as } r \rightarrow 0
$$

We also need to deduce the behavior of the various functions at the surface of the star. We must implement the usual junction conditions [35] for the spacetime functions at $r$ $=R$, and also impose a boundary condition on the fluid variable $H$.

The surface of the star is formally defined by the vanishing of the Lagrangian variation in the pressure $\Delta p$. That is, at the surface we have

$$
\Delta p=\delta p+\xi^{r} \frac{d p}{d r}=0
$$

This immediately leads to $\delta p \sim \rho \xi^{r}$ as $r \rightarrow R$, or

$$
H \sim \xi^{r}, \text { as } r \rightarrow R
$$

In principle, this constitutes a boundary condition for $H$ at the surface of the star, but in practice this result is not very helpful. First of all, we are not explicitly calculating $\xi^{r}$. Moreover, it is known from Newtonian pulsation theory that $\xi^{r}$ is not constrained at the surface of the star (it need only be non-singular). Thus, the vanishing of $\Delta p$ as $r \rightarrow R$ does not lead to a useful boundary condition for the fluid variable $H$. Instead, we infer the behavior of $H$ at $r=R$ from Eq. (16). However, because the sound speed $C_{s}$ vanishes as $r \rightarrow R$, it is not convenient to use Eq. (16) close to the surface. The behavior of $H$ at the stellar surface follows after we take the limit $C_{s} \rightarrow 0$ in Eq. (16). Keeping only terms of order $1 / C_{s}^{2}$, we get

$$
\begin{array}{r}
\ddot{H}+\frac{M}{R^{2}} \frac{\partial H}{\partial r_{*}}-\frac{M}{2 R^{3}} \frac{\partial F}{\partial r_{*}}+\frac{M}{2(R-2 M)} \frac{\partial S}{\partial r_{*}} \\
+\frac{M(R+2 M)}{2 R^{5}} F+\frac{M}{2 R(R-2 M)} S=0,
\end{array}
$$

at $r=R$. We use this equation to evolve $H$ at the surface of the star.

Let us now consider the behavior of the two "metric", variables at $r=R$. From the junction conditions (the continuity of the first and second fundamental forms across the surface of the star [25]) we find that $F, S$, and $\partial S / \partial r_{*}$ are continuous at $r=R$. That is, if we let subscripts $i$ and $e$ represent functions obtained for the interior and the exterior, respectively, we have

$$
F_{i}=F_{e}, \quad S_{i}=S_{e}, \quad \frac{\partial S_{i}}{\partial r_{*}}=\frac{\partial S_{e}}{\partial r_{*}} .
$$

It is also apparent from Eq. (14) that these conditions imply

$$
\frac{\partial^{2} S_{i}}{\partial r_{*}^{2}}=\frac{\partial^{2} S_{e}}{\partial r_{*}^{2}}
$$

as long as $\rho \rightarrow 0$ as $r \rightarrow R$. This means that all terms in Eq. (14) are well defined at the boundary, and this equation can consequently be used to evolve $S$ there.

For the function $F$ the situation is somewhat different. First we notice that we can derive an equation similar to Eq. (27) also for $F$ (i.e., an equation with no second derivative with respect to $\left.r_{*}\right)$. This equation is

$$
\ddot{F}-\frac{M}{R^{2}} \frac{\partial F}{\partial r_{*}}-R \frac{\partial S}{\partial r_{*}}-\frac{3 M}{R^{3}}\left(1-\frac{2 M}{R}\right) F-(n+1) S=0,
$$

at $r=R$. Using this equation, and the already established continuity of $\partial S / \partial r_{*}$, we can infer that

$$
\frac{\partial F_{i}}{\partial r_{*}}=\frac{\partial F_{e}}{\partial r_{*}}
$$

Thus, $\partial F / \partial r_{*}$ is continuous across the surface of the star.

Finally, it follows from the wave equation (15) that 


$$
\frac{\partial^{2} F_{i}}{\partial r_{*}^{2}}-\frac{\partial^{2} F_{e}}{\partial r_{*}^{2}}=8 \pi R e^{\nu(R)} H(R) \lim _{r \rightarrow R} \frac{p+\rho}{C_{s}^{2}} \rightarrow 8 \pi R e^{\nu(R)} H(R) \frac{\rho^{2-\gamma}}{\kappa \gamma} \quad \text { as } \quad r \rightarrow R,
$$

where $\kappa$ and $\gamma$ are the constant and index in the polytropic equation of state, respectively. Thus we see that in the case that we consider $(\gamma=2)$ the right hand side of Eq. (32) approaches a constant as $r \rightarrow R$. Consequently, the second derivative of $F$ will be discontinuous across the surface of the star.

\section{TWO MODEL PROBLEMS}

In the previous section we discussed the equations that govern perturbations of a relativistic star. We now want to evolve these equations from a given set of initial data. The ultimate purpose of this exercise is to use the perturbation approach to infer what one should expect in a problem of physical interest, e.g., when two neutron stars coalesce. At the present time such discussions are beyond our means. Before trying to implement "astrophysically relevant" initial data, we must ensure that we can evolve the stellar perturbation equations accurately. Hence, we have tested our evolution code by experimenting with different kinds of initial data. This enables us to conclude that our numerical implementation of the various equations is reliable. We also gain some insight into the excitation of the stellar pulsation modes.

\section{A. Specifying initial data}

To define acceptable initial data for the evolution problem is not a trivial task. To specify astrophysically relevant initial data, one should first solve the fully nonlinear threedimensional initial-value problem for (say) a newly formed neutron star that settles down after core collapse. Then the results must be translated into a form that makes them useful as initial data for the perturbation equations. Although easy to describe in words, each step is difficult and requires great care (cf. the analogous problem of two colliding black holes [13]). In short, a detailed formulation of initial data for the perturbation equations requires much further work, and we will return to it in the future. Here we will focus on the evolution of the perturbation equations from initial data that seems logical.

A careful analysis of the constraints that our initial data must satisfy indicates that we are free to choose our evolved variables $\{F, S, H\}$ such that the Hamiltonian constraint and its first derivative are satisfied (of course all boundary conditions and physical constraints must also be satisfied) [36]. As an initial simplification we consider only time-symmetric initial data $(\dot{F}=\dot{S}=\dot{H}=0)$. This assumption leads to rather contrived initial data from a physical point of view. Basically, the waveforms that we see as outgoing at future null infinity were initially incoming at past null infinity. Nevertheless, time-symmetric initial data provide a useful starting point for studies of the evolution problem. It should also be pointed out that the time derivative of the Hamiltonian constraint $\dot{\mathcal{H}}=0$ is automatically satisfied for time-symmetric data.

The choice of $\{F, S, H\}$ to satisfy $\mathcal{H}=0$ on the initial hypersurface depends on the model problem under consideration. So far we have considered two different classes of data sets. The first involves no initial fluid perturbation in the star, while the second includes a nonzero fluid perturbation.

The first initial data set corresponds to the scattering of an incoming gravitational wave packet by the star. Here the fluid variable $H$ is set to zero, and the metric variables initially have support only in the exterior vacuum. To enable comparison with the axial problem considered by Andersson and Kokkotas [6] we specify the Zerilli function, $Z$ to be a narrow Gaussian centered at a large radius. The spacetime variable $F$ can then be calculated using

$$
F=r \frac{\partial Z}{\partial r_{*}}+\frac{n(n+1) r^{2}+3 n M r+6 M^{2}}{r(n r+3 M)} Z .
$$

This equation follows when the definition (19) of the Zerilli function is combined with the constraint equation (17). Finally, we specify the remaining function $S$ by numerically integrating the constraint equation $\mathcal{H}=0$. An example of such initial data is shown in Fig. 1.

The scattering of gravitational waves is, however, not the problem that we are interested in. Generically, one would expect a non-vanishing perturbation inside the star. Thus, we would like to consider various perturbations in the stellar fluid. That is, we specify $H$ (arbitrarily) at some initial time. From the Hamiltonian constraint, it is clearly seen that such a fluid perturbation must be accompanied by a non-vanishing metric perturbation. Thus, we should formally find $S$ and $F$ in such a way that the constraint equation (17) is satisfied inside the star. There is no a priori method of calculating $S$ and $F$ uniquely, since we seemingly have the freedom to choose either of these two functions. To test our numerical code, we have therefore made calculations for three different kinds of initial data:

(1) We combine the specified function $H$ with $F=0$. This is convenient since we then only need to integrate a first order differential equation (17) to determine the corresponding $S$.

(2) An alternative to this is inspired by the weak-field limit. It is known that (in the standard Regge-Wheeler notation) $H_{0}-K \rightarrow 0$ as the star becomes Newtonian [37]. This would correspond to $S=0$. Once we have made this assumption, we can numerically integrate the second order differential equation (17) and find an acceptable $F$.

(3) The third set of initial data is also based on the weak 


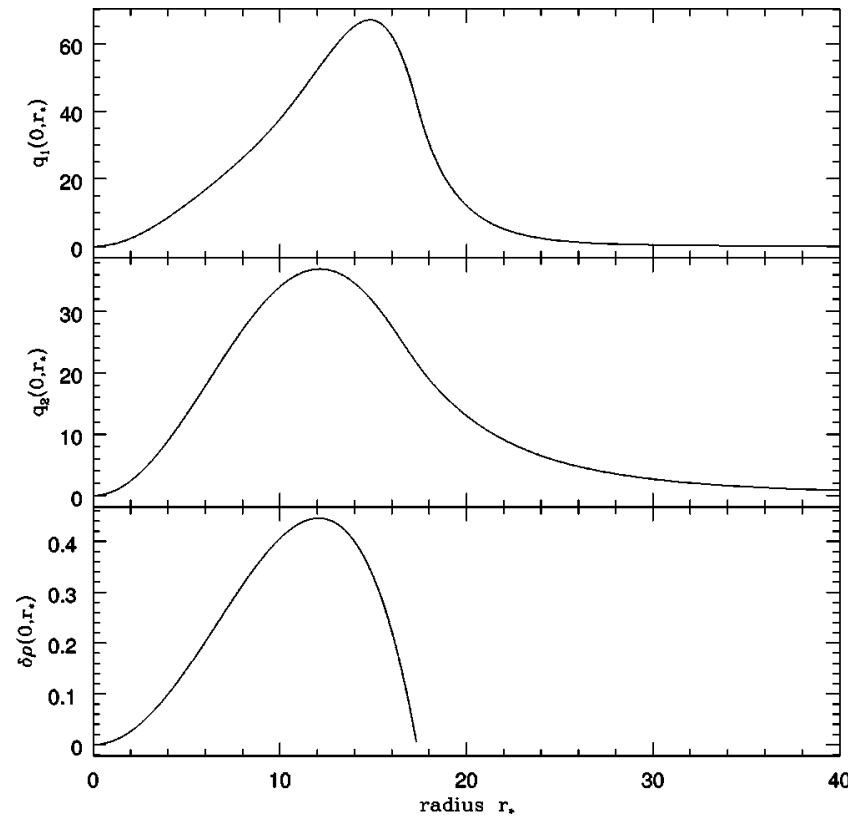

FIG. 1. Initial data for the case when an initial perturbation in the fluid is specified. Here we use $H=A C_{s}^{2}(r / R)^{2} \cos (\pi r / 2 R)$ and solve the constraint equations to obtain the required $S$ and $F$. These then lead to the two gauge-invariant quantities $q_{1}$ and $q_{2}$ (defined in Sec. II C) shown here.

field result. We set $F=2 \delta U$, where $\delta U$ is the perturbation in the Newtonian potential. Then $\delta U$ satisfies [37]

$$
\nabla^{2} \delta U=-4 \pi \delta \rho=-\frac{4 \pi(p+\rho)}{C_{s}^{2}} H
$$

Then the appropriate function $S$ is obtained by integrating Eq. (17). An example of this kind of initial data, for

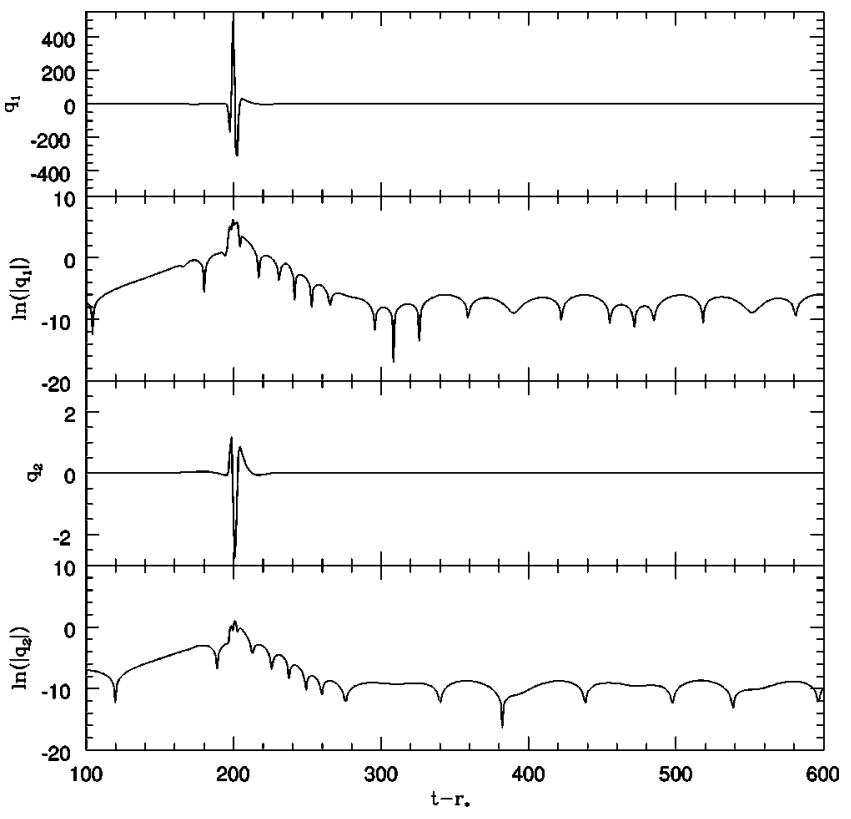

FIG. 3. Evolution of the gauge-invariant quantities $q_{1}$ and $q_{2}$ for initial data corresponding to a Gaussian Zerilli function, cf. Fig. 2.

$$
H=C_{s}^{2}(r / R)^{2} \cos (\pi r / 2 R),
$$

is shown in Fig. 1.

\section{B. Numerical results}

Even though the initial data for the model scenarios that we have chosen (see Figs. 1 and 2) are rather different, the gravitational waves that emerge from the system during each evolution are qualitatively quite similar.

In Fig. 3 we show the gravitational waves that follow when a Gaussian in the Zerilli function is scattered off the star. The corresponding initial data is shown in Fig. 2. We use the gauge invariant quantities $q_{1}$ and $q_{2}$ [cf. (23)] to represent the gravitational waves that reach a distant observer. In this specific example, the observer is located at $r_{*}=200 M$. After a sudden burst of waves follows a ring-

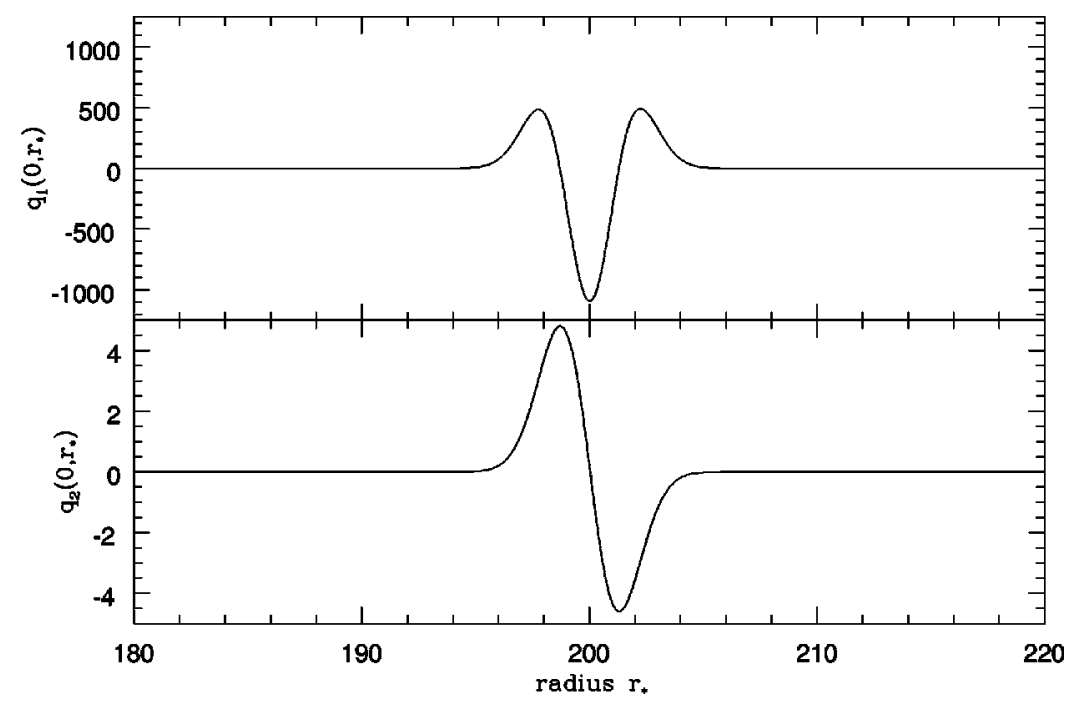

FIG. 2. Initial data corresponding to a Gaussian pulse in the Zerilli function. We show the two gauge-invariant quantities $q_{1}$ and $q_{2}$ (defined in Sec. II C). 


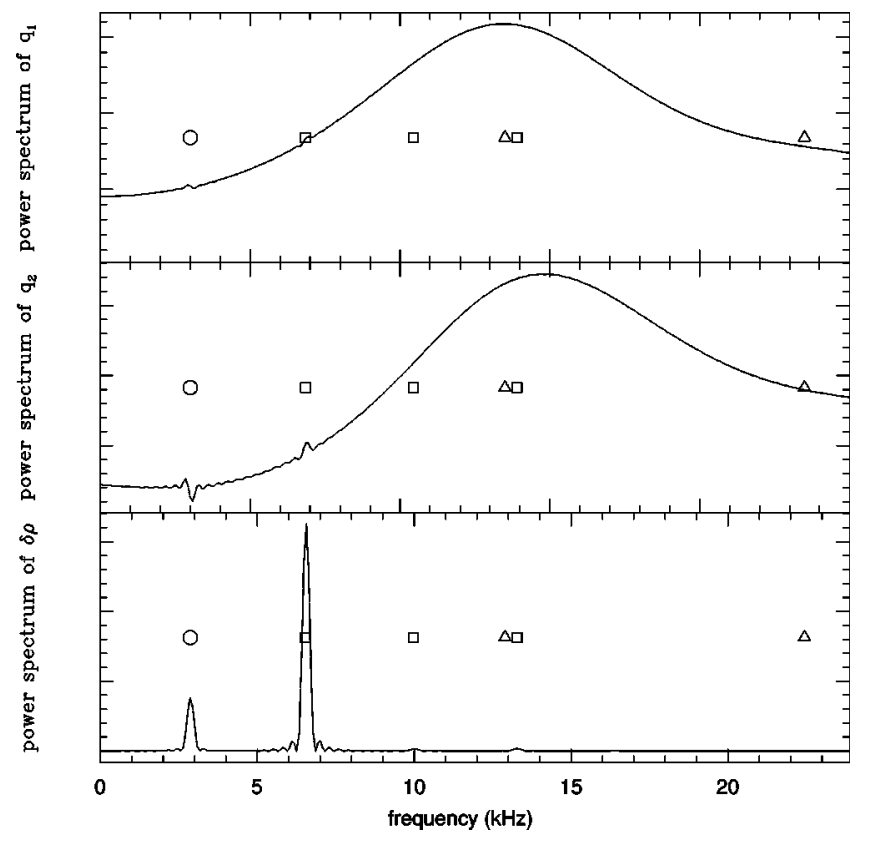

FIG. 4. Power spectrum for the data in Fig. 3. The part of the signal that is used is shown in the upper right-hand corner of each panel. The two upper panels show the power spectra for the gaugeinvariant quantities $q_{1}$ and $q_{2}$, respectively. The lower panel shows the power spectrum for the fluid motion (as represented by the Eulerian variation in the density $\delta \rho$ ). In each panel we also show the position of the various pulsation modes of the star. The $f$-mode is represented by a circle, the $p$-modes by squares, and the $w$-modes by triangles. It is clear that the emerging gravitational waves contain the first $w$-mode together with the $f$-mode and the first $p$-mode.

down corresponding to the quasinormal modes of the star. The ringdown consists of two parts. The first part (from $t$ $-r_{*} \approx 200-280 M$ in Fig. 3) shows the high frequencies and the rapid damping that are characteristics of the gravitational-wave modes. The second part of the signal (for $\left.t-r_{*}>280 M\right)$ is slowly damped, and the oscillations have longer wavelength. This part of the signal should correspond to the fluid pulsation modes. A spectral analysis of the signal shows that the emerging waves are composed of the $f$-mode, the first $p$-mode and the slowest damped $w$-mode for the star, cf. Fig. 4.

The result is similar in the case when the stellar fluid is perturbed initially. An example of such an evolution is shown in Fig. 5. This example corresponds to the initial data (35), cf. Fig. 1. Again, the post-burst signal can be roughly decomposed into two parts: the first part $\left(t-r_{*} \approx 0-25 M\right)$ corresponds to the $w$-modes and the later signal $\left(t-r_{*}\right.$ $>25 M)$ is due to the fluid modes. The corresponding spectrum shows the presence of the $f$-mode, the first $p$-mode and the slowest damped $w$-mode, cf. Fig. 6 .

These two examples show that one should expect both fluid and gravitational pulsation modes to be excited in a generic situation. But it is also clear that the relative amplitudes of the various modes depend on the initial data. In the case of a gravitational-wave pulse impinging on the star (Figs. 3 and 4) more energy is released through the $w$-modes than in the case of an initially perturbed fluid (Figs. 5 and 6).

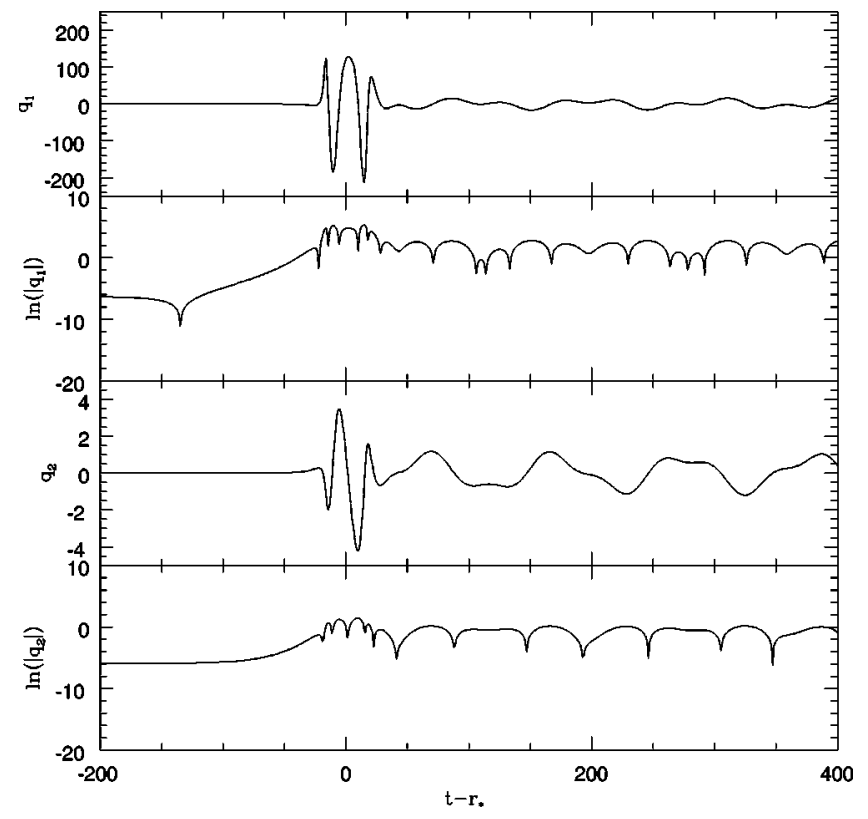

FIG. 5. Evolution of the gauge-invariant quantities $q_{1}$ and $q_{2}$ for the initial data shown in Fig. 1.

This is not very surprising, since in the first case, the impinging waves must excite motion in the fluid, which then leads to gravitational waves with (say) $f$-mode characteristics. In the second case, the fluid is already perturbed so one would expect a more pronounced excitation of the fluid modes.

Our results indicate that there can be situations where a considerable amount of energy is released through the $w$-modes, but one should be careful not to read too much physics into this result. One reason for caution is that we find that the three ad hoc ways to specify initial data with an initial fluid perturbation (see the previous section) lead to different predictions for the energy released through $w$-modes. Basically, we find that the excitation of the $w$-modes increases with the initial value of $S$. That is, if we set $S=0$, the evolutions only show a glimmer of $w$-mode oscillations. In contrast, the $w$-modes dominate the signal in cases, where $F=0$ initially. The answer to the question whether the $w$-modes will be excited to a detectable level in an astrophysically relevant situation requires a more detailed study.

\section{A brief survey of the literature}

Since we have found that several of the stars pulsation modes are excited in our test simulations, it is worthwhile to compare our results to previous numerical neutron star simulations. The available simulations fall into two categories: Simulations of coalescing stars and studies of rotating core collapse.

Almost uniquely, studies of coalescing neutron stars use Newtonian hydrodynamics and extract the gravitational waves via the quadrupole formula. As already mentioned above, this approach cannot account for the $w$-modes, but should be able to reveal the presence of the fluid pulsation modes-provided that these are excited to a significant level. A survey of the literature reveals several indications that the 


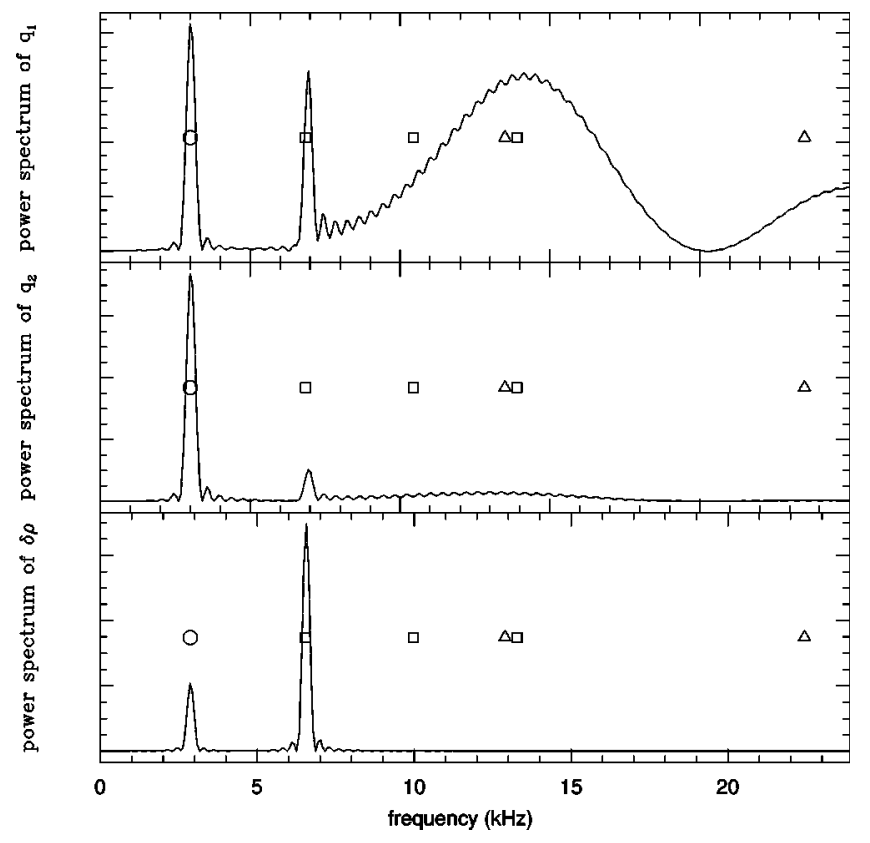

FIG. 6. Power spectrum for the data in Fig. 5. The part of the signal that is used is shown in the upper right-hand corner of each panel. The two upper panels show the power spectra for the gaugeinvariant quantities $q_{1}$ and $q_{2}$, respectively. The lower panel shows the power spectrum for the fluid motion (as represented by the Eulerian variation in the density $\delta \rho$ ). In each panel we also show the position of the various pulsation modes of the star. The $f$-mode is represented by a circle, the $p$-modes by squares, and the $w$-modes by triangles. It is clear that the emerging gravitational waves contain the first $w$-mode together with the $f$-mode and the first $p$-mode.

pulsation modes are present in the gravitational wave signals from coalescing stars. Waveforms obtained by Nakamura and Oohara [38] show clear mode presence (cf. Figs. 5, 8 and 11 in [38]). Their Fig. 5 is actually very similar to our Fig. 5. Ruffert et al. [39] have also obtained gravitational wave signals from coalescing stars that show late-time oscillations. Their waveforms and spectra (cf. Figs. 24 and 27 in [39]) show oscillations at frequencies between $1.5-2 \mathrm{kHz}$.

Further possible evidence can be found in the results of Zhuge et al. [40,41]. They model coalescing polytropic stars using SPH. In their waveforms (cf. Fig. 6 in [40]) one can see transient oscillations in the late-time waveforms. However, even though all the calculated spectra show a clear peak beyond the cutoff frequency that is associated with the actual coalescence (cf. Fig. 12 in [40]), one must be careful and not assign these oscillations too much physical relevance. Although the oscillations could correspond to certain pulsation modes (possibly $p$-modes) in the merged objects, the oscillations are tiny and their true nature is likely to be strongly compromised by numerical effects (especially the artificial viscosity) [41]. Nevertheless, the indicated oscillations are suggestive and future studies, with better resolution, should be able to provide a reliable physical interpretation.

The situation is similar as far as rotating core collapse is concerned. Most available studies use Newtonian hydrodynamics and account for gravitational-wave emission through the quadrupole formula. The collapse of a nonrotating star is expected to bounce at nuclear densities, but if the star is rotating, the collapse can also bounce at subnuclear densities because of the centrifugal force. In each case, the emerging gravitational waves are dominated by a burst associated with the bounce. But the waves that follow a centrifugal bounce can also show large amplitude oscillations that may be associated with pulsations in the collapsed core. Such results have been obtained by Mönchmeyer et al. [42]. Some of their models show the presence of modes with different angular dependence superimposed (see Fig. 5 in [42]). Typically, these oscillations have a period of a few $\mathrm{ms}$ and damp out in $20 \mathrm{~ms}$. The calculations also show that the energy in the higher multipoles is roughly three orders of magnitude smaller than that of the quadrupole. More recent simulations by Yamada and Sato [43] and Zwerger and Müller [44] also show post-bounce oscillations (cf. waveforms in Figs. 2-4 of [43] and Figs. 5 and 6 of [44]).

There is thus some indications that the fluid pulsation modes of a star will be excited in both the collapse and the coalescence scenario [45]. But it is not a clear-cut case: The oscillations that are seen are often tiny, and may be due to spurious numerical effects rather than physics. However, the cited examples are encouraging, and it seems reasonable that the $w$-modes should also be excited in a generic case. But to show that this is the case one must incorporate general relativity in the simulations of collapse and coalescence. As yet there has been few attempts to do this, but an interesting example is provided by the core-collapse studies of Seidel et al. [27,26,28]. The approach adopted in those papers is, in fact, quite similar to our present one: One considers axial (odd parity) or polar (even parity) perturbations of a timedependent background (that evolves according to a specified collapse scenario). As expected from the Newtonian studies, the extracted gravitational waves are dominated by a sharp burst associated with the bounce at nuclear densities. But there are also features that may be related to the $w$-modes. In a case designed to collapse and rebound with extreme energy Seidel et al. find a ringing mode in the post-bounce gravitational waves (cf. Fig. 7(b) of [26]). Since there are no axial fluid modes for a nonrotating star, it is plausible that this mode corresponds to one of the axial $w$-modes of the core. Furthermore, the power spectrum for one of the simulations discussed in [27] (cf. their Fig. 2) shows some enhancement around $7 \mathrm{kHz}$. As the authors state, this "is probably a result of some numerical noise... rather than a true physical effect," but it may be relevant to note that the slowest damped axial $w$-mode has a pulsation frequency of roughly this magnitude.

\section{CONCLUDING DISCUSSION}

In this paper we have discussed the evolution problem for the equations that govern a linear perturbation of a relativistic star. We have taken the first steps towards developing a framework within which one can study the behavior of a neutron star at the late stages after a gravitational collapse or a binary coalescence. Such a framework would provide a powerful testbed for fully relativistic simulations. Further- 
more, we can also hope to learn some physics from the present approach.

The results we have presented suggest that the gravitational waves that are generated by a perturbed neutron star will carry the signature of both the gravitational-wave modes and the fluid pulsation modes. This is a significant result because it indicates that present estimates of the gravitational-wave emission from gravitational collapse and coalescing neutron stars (that are mainly based on Newtonian calculations and the quadrupole formula) may be flawed. Such studies would never yield the $w$-mode part of the signal, so the energy that is released through these modes would not be accounted for.

However, to show that the $w$-modes are astrophysically relevant (here we have only provided evidence that they can carry a considerable energy in certain model situations), we must consider the question of initial data more carefully. This can be done either by extending our equations to the case of gravitational collapse (allowing the background to be time dependent), or by incorporating a $t=$ constant data set from a fully relativistic 3D collapse. After extracting the perturbations, one can then evolve them on a static background. We will return to this problem in the near future.

\section{ACKNOWLEDGMENTS}

We acknowledge helpful discussions with Horst Beyer, Curt Cutler, Pablo Laguna, Lee Lindblom, Philip Papadopoulous, Bernd Schmidt and Ed Seidel. This work was supported by an exchange program from the British Council and the Greek GSRT, and N.A. was supported by NFS (Grant No. PHY 92-2290) and NASA (Grant No. NAGW 3874). N.A. and K.D.K. also thank the Albert-Einstein Institute in Potsdam for generous hospitality.

\section{APPENDIX: THE NUMERICAL EVOLUTIONS}

We discretized the evolution equations (14), (15) and (16) in a standard way, using second order centered finite differencing, on a regular grid. The grid is equally spaced in the time coordinate $t$ and the spatial coordinate $r_{*}$. Since the coefficients in the equations are functions of the Schwarzschild radius $r$, we have to solve Eq. (13) numerically.

Some of the coefficients in the evolution equations diverge at the origin, and the finite difference equations cannot be used close to $r=0$. However, as discussed in Sec. II D, the dynamical variables all approach zero as $r \rightarrow 0$, and in practice it was sufficient to use the leading order behavior (24) close to the origin.

The boundary equations (27) and (30) were used to evolve the functions $H$ and $F$ at the stellar boundary, and $S$ was evolved using the evolution equation (14). A standard outgoing wave boundary condition was implemented for $S$ and $F$ at the outer boundary of the grid. This causes some reflection from the boundary, since in both cases the potential in the wave equation is small, but not zero. These reflections were too small to be "seen" in the numerical data, but to be safe, the outer boundary was placed sufficiently far away that it could have no influence on the results.

To construct initial data the Hamiltonian constraint (17) must be solved. For the cases we considered this reduced to solving either a first order ordinary differential equation (ODE) for $S$ (when $F$ and $H$ were given) or a second order ODE for $F$ (when $S$ and $H$ were given). The first order ODE was solved using a fourth order Runge-Kutta method [46], and the second order ODE was solved using a relaxation method. A potential problem with finding the solution at the stellar boundary (where the second space derivative of $F$ can be discontinuous if $H \neq 0$, see Sec. II D) was avoided by only using initial data with $H=0$ at the boundary.

Analytically, the Hamiltonian constraint $\mathcal{H}=0$ is satisfied throughout the evolution if it is satisfied initially. Numerically, there will always be some errors, and we can calculate $\mathcal{H}$ in the discrete $L_{2}$ norm over the grid on each $t$ $=$ constant slice and plot the result as a function of time. Calculating this error using different grid resolutions for a particular model allows the convergence of the code to be tested. For all the results reported here, second order convergence was found.
[1] P. N. McDermott, H. M. Van Horn, and J. F. Scholl, Astrophys. J. 268, 837 (1983).

[2] P. N. McDermott, H. M. Van Horn, and C. J. Hansen, Astrophys. J. 325, 725 (1988).

[3] A. Gautschy and H. Saio, Annu. Rev. Astron. Astrophys. 33, 75 (1995).

[4] N. Andersson, Y. Kojima, and K. D. Kokkotas, Astrophys. J. 462, 855 (1996).

[5] K. D. Kokkotas and B. F. Schutz, Mon. Not. R. Astron. Soc. 255, 119 (1992).

[6] N. Andersson and K. D. Kokkotas, Phys. Rev. Lett. 77, 4134 (1996).

[7] N. Andersson and K. D. Kokkotas, Mon. Not. R. Astron. Soc. (to be published).

[8] N. Andersson, in Physics of black holes, edited by I. D. No- vikov and V. Frolov (Kluwer Academic, Dordretch, 1997), Chap. 4.

[9] A. Abramovici, W. E. Althouse, R. W. P. Drever, Y. Gursel, S. Kawamura, F. J. Raab, D. Shoemaker, L. Sievers, R. E. Spero, K. S. Thorne, R. E. Vogt, R. Weiss, S. E. Whitcomb, and M. E. Zucker, Science 256, 325 (1992).

[10] C. Bradaschia, R. DelFabbro, A. DiVirgilio, A. Giazotto, H. Kautzky, V. Montelatici, D. Passuello, A. Brillet, O. Cregut, P. Hello, C. N. Man, P. T. Manh, A. Marraud, D. Shoemaker, J. Y. Vinet, F. Barone, L. Difiore, L. Milano, G. Russo, J. M. Aguirregabiria, H. Bel, J. P. Duruisseau, G. Ledenmat, P. Tourrenc, M. Capozzi, M. Longo, M. Lops, I. Pinto, G. Rotoli, T. Damour, S. Bonazzola, J. A. Marck, E. Gourghoulon, L. E. Holloway, F. Fuligni, V. Iafolla, and G. Natale, Nucl. Instrum. Methods Phys. Res. A, p. 518 (1990). 
[11] Proceedings of Astrophysical sources of gravitational radiation, Les Houches Summer School, 1995, edited by J. A. Marck and J. P. Lasota (Cambridge University Press, Cambridge, England, 1997).

[12] S. Bonnazolla and J. A. Marck, Annu. Rev. Nucl. Part. Sci. 45, 655 (1994).

[13] R. H. Price and J. Pullin, Phys. Rev. Lett. 72, 3297 (1994).

[14] K. S. Thorne and A. Campolattaro, Astrophys. J. 149, 591 (1967).

[15] K. D. Kokkotas, Pulsating relativistic stars in Proceedings of Astrophysical sources of gravitational radiation, Les Houches Summer School, 1995, edited by J. A. Marck and J. P. Lasota (Cambridge University Press, Cambridge, England, 1996).

[16] L. Lindblom and S. L. Detweiler, Astrophys. J., Suppl. Ser. 53, 73 (1983).

[17] K. D. Kokkotas and B. F. Schutz, Gen. Relativ. Gravit. 18, 913 (1986).

[18] N. Andersson, K. D. Kokkotas, and B. F. Schutz, Mon. Not. R. Astron. Soc. 280, 1230 (1996).

[19] Y. Kojima, N. Andersson, and K. D. Kokkotas, Proc. R. Soc. London 451, 341 (1995).

[20] N. Andersson, Gen. Relativ. Gravit. 28, 1433 (1996).

[21] A. Borrelli and V. Ferrari, preprint (1995).

[22] S. L. Detweiler and L. Lindblom, Astrophys. J. 292, 12 (1985).

[23] J. R. Ipser and R. H. Price, Phys. Rev. D 43, 1768 (1991).

[24] Y. Kojima, Phys. Rev. D 46, 4289 (1992).

[25] S. Kind, J. Ehlers, and B. G. Schmidt, Class. Quantum Grav. 10, 2137 (1993).

[26] E. Seidel and T. Moore, Phys. Rev. D 35, 2287 (1987).

[27] E. Seidel, E. S. Myra, and T. Moore, Phys. Rev. D 38, 2349 (1988).

[28] E. Seidel, Phys. Rev. D 42, 1884 (1990).

[29] S. Chandrasekhar, The mathematical theory of black holes (Oxford University Press, Oxford, 1983).

[30] S. Chandrasekhar and V. Ferrari, Proc. R. Soc. London A433, 423 (1991).

[31] If the angular dependence of the perturbed quantities were to be accounted for, we should use

$$
h_{\theta \theta}(t, r, \theta, \varphi)=r \sum_{l, m} F_{l m}(t, r) Y_{l m}(\theta, \varphi),
$$

etc., where $Y_{l m}(\theta, \varphi)$ are the standard spherical harmonics. The fluid variables $\xi^{i}, \delta p$ and $\delta \rho$ are, of course, also expanded in spherical harmonics. For a more detailed description of the appropriate angular functions, see $[14,24]$. Our shorthand notation should not lead to any confusion, since we focus on the perturbation functions and never actually reconstruct the perturbed metric. Furthermore, it should be noted that we only consider the quadrupole case $(l=2)$ in our numerical examples, and since the background geometry is spherically symmetric, it is sufficient to consider $m=0$.

[32] The wave equation for $S$ comes from the $[\theta-\varphi \theta-\varphi]$ component of the perturbed Einstein equations, the equation for $F$ follows when one combines the $[t t]$ and the $[r r]$ components of the perturbed Einstein equations, and the wave equation for the variable $H$ follows from the time-derivative of the $t$-component of the equations of motion for the fluid. The Hamiltonian constraint corresponds to the $[t t]$-component of the perturbed Einstein equations. For more details of the origin of all these equations, we refer to $[24,25]$.

[33] S. Chandrasekhar and V. Ferrari, Proc. R. Soc. London A433, 449 (1991)

[34] V. Moncrief, Ann. Phys. (N.Y.) 88, 343 (1974).

[35] C. W. Misner, K. S. Thorne, and J. A. Wheeler, Gravitation (W. H. Freeman, San Fransisco, 1973).

[36] Appropriate initial data must satisfy the Hamiltonian constraint (17), together with the two momentum constraints (that follow from the $[t r]$ and the $[t \theta]$ components of the perturbed Einstein equations [24]). In order for the Hamiltonian constraint to be preserved during the evolution, we must also require that the first time derivative of $\mathcal{H}$ vanishes. However, if we write the two momentum constraints schematically as

$$
\begin{aligned}
& \mathcal{M}_{r}\left(F, S, H, h_{t r}, \xi^{r}\right)=0, \\
& \mathcal{M}_{\theta}\left(F, S, H, h_{t r}, \xi^{r}\right)=0,
\end{aligned}
$$

one can show that [25]

$$
\dot{\mathcal{H}}=\mathcal{M}_{r}^{\prime}+\mathcal{M}_{r}+\mathcal{M}_{\theta} \text {. }
$$

Now, the Hamiltonian constraint and its first time derivative involves only the variables which are evolved using equations (14), (15) and (16). Namely, the set $\{F, S, H, \dot{F}, \dot{S}, \dot{H}\}$. Thus we need only require that our initial data satisfies $\mathcal{H}=\dot{\mathcal{H}}=0$. The two momentum constraints then act as definitions of the metric perturbation $h_{t r}$ and the fluid displacement $\xi^{r}$.

[37] K. S. Thorne, Astrophys. J. 158, 997 (1969).

[38] T. Nakamura and K. Oohara, Prog. Theor. Phys. 86, 73 (1991).

[39] M. Ruffert, H. T. Janka, and G. Schäfer, Astron. Astrophys. 311, 532 (1995)

[40] X. Zhuge, J. M. Centrella, and S. L. W. McMillan, Phys. Rev. D 50, 6247 (1994).

[41] X. Zhuge, J. M. Centrella, and S. L. W. McMillan, Phys. Rev. D 54, 7261 (1996).

[42] R. Mönchmeyer, G. Schäfer, E. Müller, and R. E. Kates, Astron. Astrophys. 246, 417 (1991).

[43] S. Yamada and K. Sato, Astrophys. J. 450, 245 (1995).

[44] T. Zwerger and E. Müller, Astron. Astrophys. 320, 209 (1997).

[45] It is worth pointing out that although the various results in the literature indicate that stellar pulsation modes are present in the gravitational-wave signals at late times, it is not trivial to infer exactly which the relevant modes are. For example, in the case of core collapse, a mode-analysis is complicated by the fact that the pulsating core is both rotating (most likely quite rapidly) and accreting matter. In comparison, our model evolutions are very clean.

[46] W. H. Press, B. P. Flannery, S. A. Teukolsky, and W. T. Vetterling, Numerical Recipes (Cambridge University Press, Cambridge, England, 1986). 